\title{
Endobronchial Carcinoid Tumor Totally Occluding the Left Main Bronchus Without Producing Symptoms of Bronchial Obstruction
}

\author{
DIAMANTIS I. TSILIMIGRAS ${ }^{1}$, DEMETRIOS MORIS ${ }^{2}$, IOANNIS NTANASIS-STATHOPOULOS ${ }^{1}$, \\ DAVIDE PATRINI ${ }^{3}$ and NIKOLAOS PANAGIOTOPOULOS ${ }^{3}$ \\ ${ }^{1}$ School of Medicine, National and Kapodistrian University of Athens, Athens, Greece; \\ ${ }^{2}$ Department of Surgery, Division of Surgical Oncology, \\ The Ohio State University Wexner Medical Center, Columbus, OH, U.S.A.; \\ ${ }^{3}$ Department of Thoracic Surgery, University College London Hospitals (UCLH), London, U.K.
}

\begin{abstract}
Background: Bronchial carcinoid tumors (BCTs) are rare neuroendocrine neoplasms of the lung that mainly have a central distribution. They are classified as typical and atypical, with the former variant generally conferring a more favorable survival. Central tumors are usually symptomatic with features of bronchial obstruction, whereas peripheral tumors may remain silent. Case Report: A 36-year-old woman presented to our hospital due to an episode of massive hemoptysis 5 days prior to admission. She had experienced another episode of hemoptysis 4 years before, for which a chest $x$-ray had shown no pathological findings. A new chest $x$-ray showed complete collapse of the left lung, with remarkable tracheal deviation. Computed tomography revealed a large endobronchial lesion causing occlusion of the left main bronchus and significant mediastinal shift to the left. Despite the collapse of the left lung, no symptoms of bronchial obstruction were evident. The patient underwent a successful left pneumonectomy and pathology of the resected specimen revealed a typical stage pT2b N1 Mx endobronchial carcinoid tumor. Conclusion: Central tumors are usually symptomatic with features of bronchial obstruction, whereas peripheral tumors may remain silent. Although a significant amount of bronchial occlusion may be present, symptoms of obstruction might not be apparent. Hemoptysis should
\end{abstract}

This article is freely accessible online.

Correspondence to: Diamantis I. Tsilimigras, MD, School of Medicine, National and Kapodistrian University of Athens, 75 Mikras Asias str., 11527, Athens, Greece. Tel: +30 2107716042, email: tsilidiam@gmail.com

Key Words: Pneumonectomy, mediastinal shift, tracheal deviation. always be thoroughly investigated, as it may be the only sign of a severe underlying disease.

Bronchial carcinoid tumors (BCTs) are neuroendocrine neoplasms of the lung and represent about $1-2 \%$ of all primary lung tumors. Although first classified as benign tumors, they are nowadays considered as low-grade malignant neoplasms (1). BCTs originate from Kulchitsky cells in the bronchial mucosa, neuroepithelial bodies, or pluripotential bronchial epithelial stem cells (2). Based on their pathological features, they are also classified as typical and atypical, with the former variant generally conferring a more favorable survival (3). About one-third of patients are asymptomatic, whereas cough, recurrent pneumonia and hemoptysis account for the majority of the symptoms, especially in the case of centrally located tumors (4). Herein, we report a case of a large BCT totally occluding the left main bronchus without producing symptoms of bronchial obstruction.

\section{Case Report}

A 36-year-old woman presented to our hospital due to an episode of massive hemoptysis 5 days prior to admission. She was a cannabis smoker and her past medical history was only remarkable for an episode of hemoptysis 4 years before, for which a chest $\mathrm{x}$-ray was performed, but no pathological findings were shown. Therefore, it was considered as incidental and no further investigation was recommended at that time. However, a new chest x-ray showed complete collapse of the left lung, with remarkable tracheal deviation (Figure 1A). Computed tomography revealed a large endobronchial lesion causing occlusion of the left main bronchus (Figure 1B) and significant mediastinal shift to the left (Figure 1C and D). Despite the collapse of the collapse 

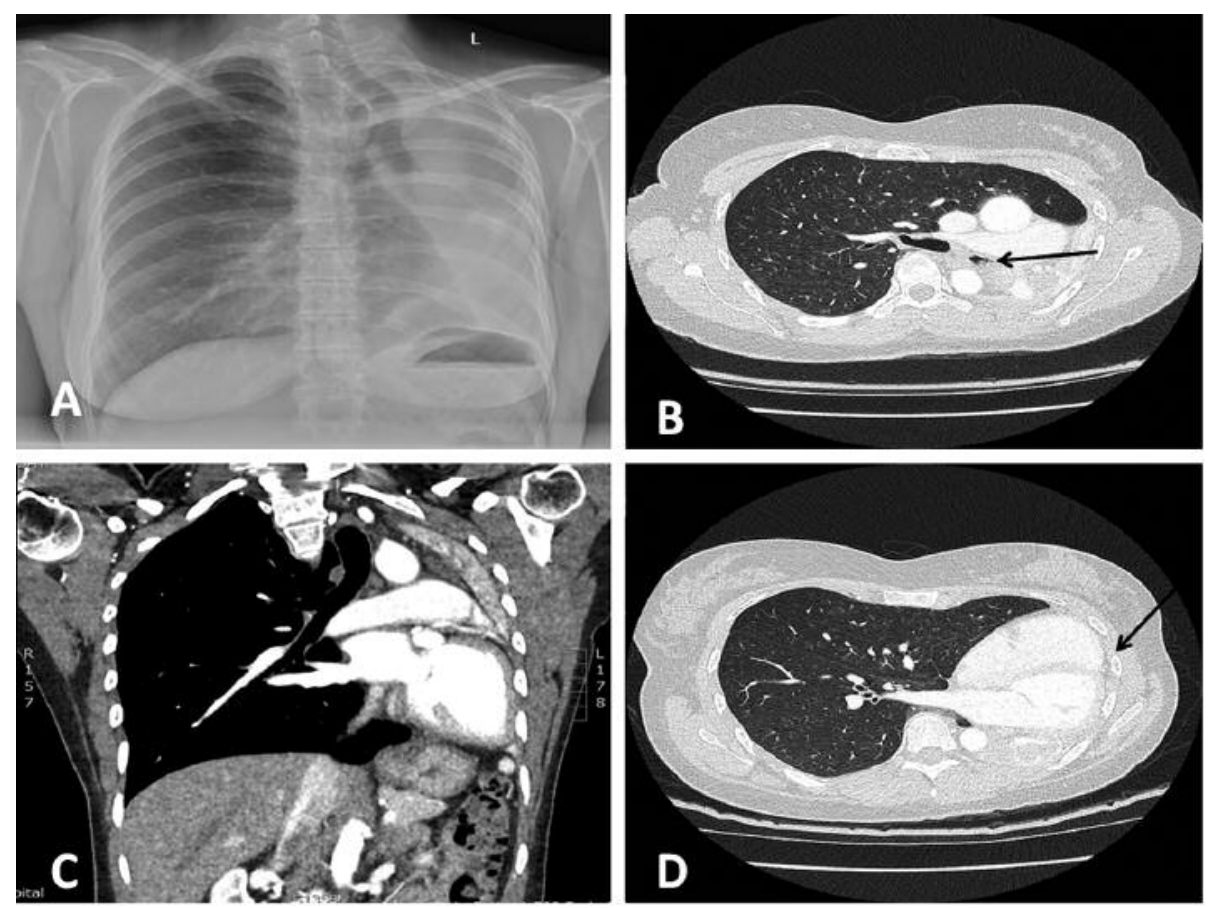

Figure 1. A: Chest x-ray showing left lung atelectasis with remarkable tracheal deviation. B: Computed tomography showing a 59-mm endobronchial lesion (arrow) causing occlusion of the left main bronchus. C: Coronal computed tomographic view of the chest. D: Significant mediastinal shift to the left, leading to the heart being in contact with the lateral chest wall (arrow).

left lung, no symptoms of bronchial obstruction were evident. The patient underwent successful left pneumonectomy through thoracotomy and pathology of the resected specimen revealed a typical stage pT2b N1 Mx endobronchial carcinoid tumor. At a follow-up of 12 months, she remains alive and all examinations have failed to reveal any recurrent disease.

\section{Discussion}

BCTs are rare neoplasms, with a reported annual incidence of $0.2-3$ per 100,000 people $(1,3)$. No clear sexual or racial predilection has been documented. Typical BCTs have been reported in all age groups but a peak around the fourth decade has been documented $(1,3)$. On the contrary, the atypical variant occurs more frequently during the fifth decade of life (1). BCTs are capable of synthesizing, storing, and secreting peptide hormones, as well as serotonin, adrenocorticotropic hormone, somatostatin and bradykinin $(4,5)$.

The vast majority of BCTs arise centrally in the main, lobar or segmental bronchi and demonstrate no specific lobar distribution (6). Central tumors are usually symptomatic with features of bronchial obstruction and ball-valve effect for polypoid tumors, whereas peripheral tumors are usually asymptomatic (6). The main complaints include cough, fever, expectoration, wheezing, hemoptysis, chest pain or symptoms simulating asthma $(7,8)$. Due to the high tumor vascularity, hemoptysis occurs in at least $50 \%$ of patients $(8$, 9 ), whereas approximately one-fourth of patients are diagnosed incidentally (9). In our case, despite the total occlusion of the left main bronchus leading to collapse of the left lung, the patient had no symptoms of bronchial obstruction, but only two episodes of hemoptysis with a time interval of 4 years between them.

Due to its rarity and the low index of suspicion, diagnosis is often missed or delayed even in symptomatic patients (1, 4). It is, therefore, of paramount importance to evaluate and examine in detail every episode of hemoptysis rather than considering it as an incidental finding. In our case, notably, if a thorough investigation had been performed at the first episode, the patient could probably have avoided pneumonectomy.

In contrast to other types of lung cancer, BCTs generally confer a better prognosis. In particular, typical BCTs are associated with superior survival compared to the atypical variant, with 5- and 10-year survival rate of 91-95\% and 86$89 \%$, respectively $(1,3)$. Furthermore, atypical BCTs have been identified as a risk factor for recurrence $(10,11)$. Fortunately, our patient was diagnosed with a typical BCT and remains healthy 12 months after pneumonectomy. 


\section{Conclusion}

BCTs are rare, low-grade malignant tumors of the lung that mainly have a central distribution. Central tumors are usually symptomatic with features of bronchial obstruction, whereas peripheral tumors may remain silent. Although a significant amount of bronchial occlusion may be present, symptoms of obstruction might not be apparent. Hemoptysis should always be thoroughly investigated, as it may be the only sign of a severe underlying disease.

\section{Conflicts of Interest}

The Authors declare no conflicts of interest.

\section{References}

1 Detterbeck FC: Management of carcinoid tumors. Ann Thorac Surg 89(3): 998-1005, 2010

2 Paladugu RR, Benfield JR, Pak HY, Ross RK and Teplitz RL: Bronchopulmonary Kulchitzky cell carcinomas. A new classification scheme for typical and atypical carcinoids. Cancer 55(6): 1303-1311, 1985.

3 Filosso PL, Guerrera F, Evangelista A, Welter S, Thomas P, Casado PM, Rendina EA, Venuta F, Ampollini L, Brunelli A, Stella F, Nosotti M, Raveglia F, Larocca V, Rena O, Margaritora S, Ardissone F, Travis WD, Sarkaria I, Sagan D and ESTS NETWG Committee: Prognostic model of survival for typical bronchial carcinoid tumours: analysis of 1109 patients on behalf of the European Association of Thoracic Surgeons (ESTS) Neuroendocrine Tumours Working Group. Eur J Cardiothorac Surg 48(3): 441-447, 2015.
4 Warren WH, Faber LP and Gould VE: Neuroendocrine neoplasms of the lung. A clinicopathologic update. J Thorac Cardiovasc Surg 98(3): 321-332, 1989.

5 Shalet SM, Beardwell CG, MacFarlane IA, Ellison ML, Norman CM, Rees LH and Hughes M: Acromegaly due to production of a growth hormone releasing factor by a bronchial carcinoid tumor. Clin Endocrinol 10(1): 61-67, 1979.

6 Nessi R, Basso Ricci P, Basso Ricci S, Bosco M, Blanc M and Uslenghi C: Bronchial carcinoid tumors: radiologic observations in 49 cases. J Thorac Imaging 6(2): 47-53, 1991.

7 Todd TR, Cooper JD, Weissberg D, Delarue NC and Pearson FG: Bronchial carcinoid tumors: twenty years' experience. J Thorac Cardiovasc Surg 79(4): 532-536, 1980.

8 Dusmet ME and McKneally MF: Pulmonary and thymic carcinoid tumors. World J Surg 20(2): 189-195, 1996.

9 Ducrocq X, Thomas P, Massard G, Barsotti P, Giudicelli R, Fuentes $\mathrm{P}$ and Wihlm JM: Operative risk and prognostic factors of typical bronchial carcinoid tumors. Ann Thorac Surg 65(5): 1410-1414, 1998.

10 Lee PC, Osakwe NC, Narula N, Port JL, Paul S, Stiles BM, Andrews WG, Nasar A and Altorki NK: Predictors of diseasefree survival and recurrence in patients with resected bronchial carcinoid tumors. Thorac Cardiovasc Surg 64(2): 159-165, 2016.

11 Cardillo G, Sera F, Di Martino M, Graziano P, Giunti R, Carbone L, Facciolo F and Martelli M: Bronchial carcinoid tumors: nodal status and long-term survival after resection. Ann Thorac Surg 77(5): 1781-1785, 2004.

Received June 11, 2017

Revised June 21, 2017

Accepted July 3, 2017 